\title{
Editorial for Special Issue on Human-inspired Computing
}

Human-inspired computing is the intelligence computing model enlightened by human brain intelligence and biological processes. It is not only the inheritance and development of artificial intelligence, but also from a new point to understand and grasp the intelligent intrinsic. With the recent advances in cognitive science, we now have the ability to mine more details of the human brain to obtain valuable insight about what is happening in the brain. Enlightened from these new achievements in the brain cognition, scientists have proposed many new theories and algorithms of human-inspired computing, which have been applied widely in many fields, such as artificial intelligence, machine learning and data mining. Human-inspired computing is becoming a significant force to promote science and technology progress.

The goal of this special issue is to call for a coordinated effort to understand the opportunities and challenges emerging in human-inspired computing, identify key tasks and evaluate the state of the art, showcase innovative methodologies and ideas, as well as introduce real systems or applications, and discuss future directions. Intelligence computing plays an important role in public security, entertainment, healthcare, etc. We solicit manuscripts in the important fields of intelligence computing that explore the synergy of human-inspired computing and deep learning techniques. In the end, six papers are accepted to this special section.

The first paper, entitled "Why and when can deep-but not shallow-networks avoid the curse of dimensionality: a review" from Tomaso Poggio et al., reviewed and extended an emerging body of theoretical results on deep learning. By comparing shallow (one-hidden layer) networks with deep convolutional networks, the authors investigated the conditions under which deep networks can be exponentially better than shallow learning. Conclusion was drawn that deep networks have the theoretical guarantee to avoid the curse of dimensionality for compositional functions, such as locality of hierarchy and compositional input-output mapping.

The second paper, entitled "Imitating the brain with neurocomputer - a "new" way towards artificial general intelligence" from Tie-Jun Huang proposed a practical approach to achieve artificial general intelligence by imitating the connection structure of brain to build up neurocomputer, based

Editorial

Special Issue on Human-inspired Computing

Manuscript received July 11, 2017; accepted July 18, 2017

(C) Institute of Automation, Chinese Academy of Sciences and Springer-Verlag GmbH Germany 2017 on the imitational philosophy. The authors provided abundant evidence to demonstrate the philosophy that "function comes from structure". By trimming the development history of artificial intelligence, and summarizing the status and tendency of neurocomputer, the authors investigated a key problem that why neurocomputer can make artificial general intelligence come true.

The third paper, entitled "Why deep neural nets cannot ever match biological intelligence and what to do about it?" from Danko Nikolić compared the differences between biological intelligence and artificial intelligent agent in adaptive capacity based on practopoiesis theory. By calculating the variety of real life, human agent and artificial agent, the author analyzed the reason why artificial intelligence (AI) at present cannot match biological intelligence and gave some advice to improve it.

The fourth paper, entitled "On the centre of mass motion in human walking" from Jean-Paul Laumond et al., proposed a new model to estimate the motion of Center of Mass (CoM). Based on the analysis about observability of human walking, the authors demonstrated that CoM follows a curtate cycloid in sagittal plane and then establish a curtate cycloid model to describe this pattern. Compared with existing approaches, it is a segmentation-free model with higher accuracy and fewer parameters. Furthermore, this proposed model potentially becomes an alternative humaninspired control approach of humanoid robotics.

The fifth paper, entitled "Flexible robotic grasping strategy with constrained region in environment" from Hong Qiao et al., investigated the brain inspired grasping strategy for dexterous robotic hands. Inspired by the grasping operation of human, the authors introduced a novel concept of constrained region in environment, which integrates a bio-inspired co-sensing framework. By utilizing the constrained region in environment, the grasping can be effectively accomplished with relatively low-precision sensors for the robots. The effectiveness of the proposed grasping strategy on constrained region in environment has been demonstrated on Barrett Hand during experiment.

The sixth paper, entitled "A brain-inspired SLAM system based on ORB features" from Huajin Tang et al., described a brain-inspired simultaneously localization and mapping (SLAM) system, dubbed RatSLAM, which is different from the existing RatSLAM system. The proposed system only uses a simple vector to represent features of visual image. This paper employs an efficient as well as fast descriptor method, called ORB, to extract features from RGB images and has demonstrated the efficacy and robust- 
ness on the dubbed RatSLAM.

$$
\begin{array}{r}
\text { Hong Qiao } \\
\text { Institute of Automation, Chinese Academy of Sciences, China } \\
\text { Hong Zhang } \\
\text { University of Alberta, Canada } \\
\text { Florian Röhrbein } \\
\text { Technical University of Munich, Germany }
\end{array}
$$

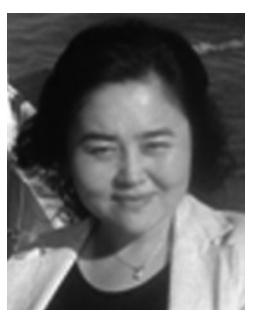

Hong Qiao is a professor with Institute of Automation, Chinese Academy of Sciences (CASIA), China. In 2004, Professor Qiao gave up the tenure position and returned to China under the grant of the "100 Talent" Program of the Chinese Academy of Sciences. After her return, she founded the "Robotic Theory and Application" group in CASIA. In 2007, she was supported by National Science Fund for Distinguished Young Scholars. Currently, she is the Core Expert of CAS Centre for Excellence in Brain Science and Intelligence Technology (CEBSIT), the vice director of Joint Lab Between the Institute of Automation and University of Sciences and Technology of China, and the deputy director \& principal investigator of Neuro-robotics, Research Centre for Brain-inspired Intelligence, Institute of Automation, CAS.

In 2014, Professor Qiao won the Second Prize of National Natural Science Awards as the first participant (this award is the highest fundamental research award in China). It is among the three ones themed in robotics in this award ever since. In 2012, Professor Qiao won the First Prize of Beijing Science and Technology Award for fundamental research as the first participant. In 2015, Professor Qiao won the Second Prize of Beijing Science and Technology Award for technology inventions as the first participant. In 2013, Professor Qiao was elected as a member of Administrative Committee, IEEE Robotics \& Automation Society (RAS), which is the first time that the mainland scholar was elected to this committee.

Professor Qiao has long led the group in research areas of robotic "Hand-Eye-Brain", which includes industrial robot manipulation and control (Hand), robot vision (Eye), bio-inspired and brain-like intelligent robot (Brain), etc. She is now serving as the Editor-in-Chief of international journal Assembly $\mathrm{Au}$ tomation, and the associate editors for several significant international journals, such as IEEE Transactions on Cybernetics,
IEEE/ASME Transactions on Mechatronics, IEEE Transactions on Automation Science and Engineering, IEEE Transactions on Cognitive and Developmental Systems.

She has undertaken a series of national key project, including National Science Fund for Distinguished Young Scholars, Key Project of Natural Science Foundation of China, 863 Project of Ministry of Science and Technology of China, "04 Project" of National Science and Technology Major Project, Project of Intelligent Equipment of National Development and Reform Commission, etc. Two industrialization bases for industrial robot have been established in Guangdong Province.

E-mail: hong.qiao@ia.ac.cn (Corresponding author) ORCID iD: 0000-0001-6384-3687

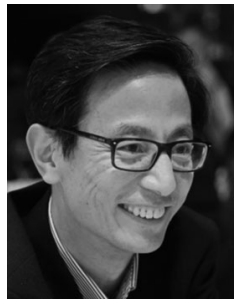

Hong Zhang is a professor with the Department of Computing Science, University of Alberta, Canada. He has held an NSERC Industrial Research Chair (IRC), supported jointly by Syncrude Canada Ltd. (SCL) and Alberta Innovates (formerly known as iCORE) since 2003. Professor Zhang is also a member of the NSERC strategic network on field robotics (NCRFN). His research interests span robotics, computer vision, and image processing. In 2017, Professor Zhang will serve as the general chair for IEEE/RSJ International Conference on Intelligent Robots and Systems (IROS) in Vancouver, Canada.

E-mail: zhang@cs.ualberta.ca

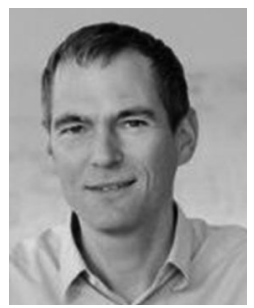

Florian Röhrbein is managing director of the Neurorobotics subproject of the Human Brain Project flagship. PD Dr. Florian Röhrbein has international work experience in various projects on braininspired cognitive systems. Previous research stays include the MacKay Institute of Communication and Neuroscience (UK), the HONDA Research Institute Europe (Germany) and the Albert Einstein College of Medicine (New York). He received his Diploma (with honors) and Ph. D. (magna cum laude) from Technical University of Munich. In 2011, he received the venia legendi for computer science from Universität Bremen. He is Chief Co-editor for Frontiers in Neurorobotics.

E-mail: florian.roehrbein@in.tum.de 